\title{
Preliminary data on the chironomid fauna of the Middle Volga region within the Republic of Tatarstan (Russia) based on hydrobiological monitoring studies
}

\author{
Tatiana A. Kondrateva ${ }^{1}$ and Larisa B. Nazarova ${ }^{2}$
}

${ }^{1}$ FPBI (Federal State Budget Institution) "Management of hydrometeorology and environmental monitoring of the Republic of Tatarstan”, Zavodskaja str., 3, 420021, Kazan. E-mail: tatjana kondrate@mail.ru ${ }^{2}$ Potsdam University, Institute for Earth and Environmental Science, Karl-Liebknecht-Str. 24-25, 14476 Potsdam-Golm. E-mail: nazarova_larisa@mail.ru

The chironomid fauna of Middle Volga within the Republic of Tatarstan, Russia, has never been a subject of a special taxonomic investigation and only larval stages of chironomids have been studied from hydrobiological samples. Taxonomic chironomid lists are known only for some water bodies of the region: Zainsk water reservoir $\left(55^{\circ} 18^{\prime} \mathrm{N} 52^{\circ} 01^{\prime} \mathrm{E}\right.$, Nazarova, 1999), Cheboksar reservoir $\left(56^{\circ} 09^{\prime} \mathrm{N} 47^{\circ} 14^{\prime} \mathrm{E}\right.$, Nazarova et al., 2004), as well as several small rivers (Yakovlev 2003; Torsuev et al. 2005; Kondrateva, Nazarova, 2011; Mingazova 2012).

Here we provide a new list of chironomid taxa from the middle section of the River Volga (within the Republic of Tatarstan, Fig.1) and its tributaries (Mesha, Kazanka, Svijaga, Steppe Zai, Vjatka, Big Cheremshan, Tojma, Avlashka, Jurashka, and small inundated reservoir of Kazan, Fig. 1). Chironomids were investigated from hydrobiological collections of zoobenthos completed by the Meteorological service of Russia (FPBI "Management of hydrometeorology and environmental monitoring of the Republic of Tatarstan"). Samples were collected between 1991 and 2014 using standard hydrobiological methods (Abakumov 1983). On large rivers (Volga, Vjatka, Big Cheremshan) samples were collected using a Petersen dredge. Small rivers were sampled using a rod dredge and hydrobiological net. The comprehensive list of chironomids collected includes 131 species from 5 subfamilies. Names of the genera and species are listed according to the Fauna Europaea catalogue: http://www.faunaeur.org/index.php.

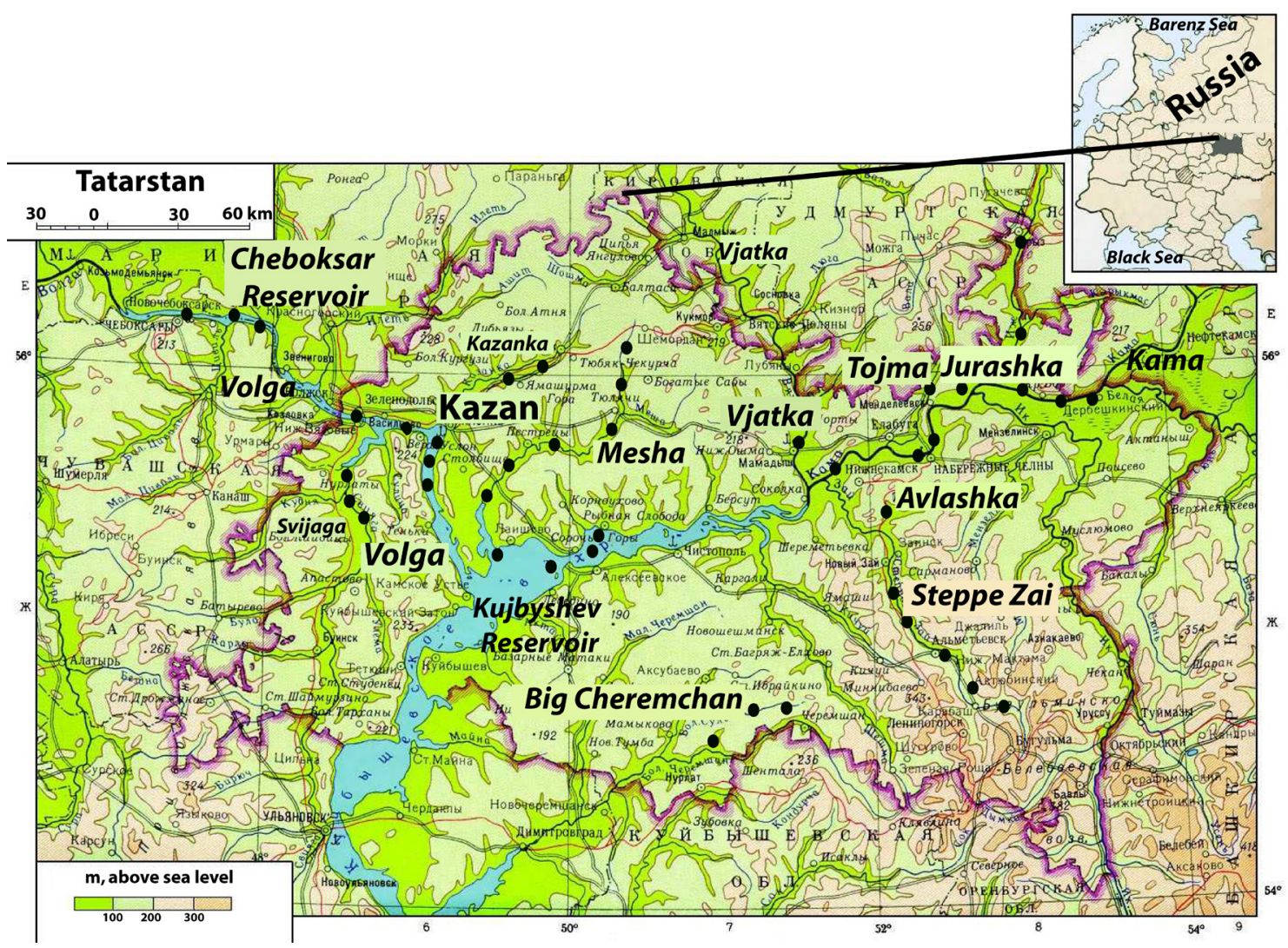

Figure 1. Map of the region. Sampling sites are given in black circles. 
Table 1. List of chironomid taxa collected from the middle stream of the River Volga (within the Republic of Tatarstan, Fig. 1) and its tributaries. Abbreviations: M - Mesha, K - Kazanka, S -Svijaga, SZ - Steppe Zai, Vj - Vjatka, BC - Big Cheremshan, T - Tojma, A - Avlashka, J - Jurashka, V - Volga, VS - small inundated reservoir of Kazan.

\begin{tabular}{|c|c|c|c|c|c|c|c|c|c|c|c|}
\hline \multirow{2}{*}{ Chironomidae collected } & \multicolumn{11}{|c|}{ Rivers } \\
\hline & $\mathrm{M}$ & $\mathrm{K}$ & $\mathrm{S}$ & SZ & $\mathrm{Vj}$ & $\mathrm{BC}$ & $\mathrm{T}$ & A & $\mathrm{J}$ & $\mathrm{V}$ & VS \\
\hline \multicolumn{12}{|l|}{ Tanypodinae } \\
\hline Ablabesmyia (Ablabesmyia) monilis & + & + & + & + & & & & & & & \\
\hline A. (Ablab.) phatta & & + & & & & & & & & & \\
\hline Ablabesmia sp. & + & + & + & & & & & & & & \\
\hline Apsectrotanypus trifascipennis & + & & & + & & & & + & & & \\
\hline Arctopelopia sp. & & & & + & & & & & + & & \\
\hline Clinotanypus pinguis & & & & & & & & & & & + \\
\hline C. nervosus & & & & & & & & & & & + \\
\hline Coelotanypus concinnus & & & & & & & & & & & + \\
\hline Conchapelopia melanops & & & & & & & & & & & + \\
\hline Conchapelopia sp. & + & & & & & & & & & & \\
\hline Guttipelopia guttipennis & & + & & & & & & & & & \\
\hline Macropelopia nebulosa & & & + & & & & & & & & \\
\hline Monopelopia tenuicalcar & & & & & & & & & & & + \\
\hline Psectrotanypus varius & & & & & & & & & & & + \\
\hline Procladius (Holotanypus) choreus & + & & & & + & & & & & & + \\
\hline P. (Holotanypus) ferrugineus & + & + & + & + & + & + & + & & & + & + \\
\hline Procladius (Holotanypus) sp. & & & + & & & & & & & & + \\
\hline Procladius (Psilotanypus) imicola & + & & & & & & & & & & \\
\hline Rheopelopia ornata & & & & + & & & & & & & \\
\hline Rheopelopia sp. & & & & + & & + & & & & + & \\
\hline Telopelopia okoboji & & & & + & & & & & & & \\
\hline Tanypus (Tanypus) kraatzi & & & & & & & & & & & + \\
\hline \multicolumn{12}{|l|}{ Diamesinae } \\
\hline Diamesa steinboecki & & & & & + & & & & & & \\
\hline Potthastia longimanus & & & & & & & & + & & + & \\
\hline P. gaedii & & & & + & & & & & & & + \\
\hline Protanypus caudatus & & & & + & & & & & & & \\
\hline \multicolumn{12}{|l|}{ Protanypus sp. } \\
\hline Pseudodiamesa (Pseudodiamesa) nivosa & & & & + & & & & & & & \\
\hline \multicolumn{12}{|l|}{ Prodiamesinae } \\
\hline Monodiamesa bathyphila & + & + & & + & & + & & & & & \\
\hline Odontomesa fulva & & & & + & & & & + & & & \\
\hline Prodiamesa olivacea & + & + & + & + & & + & + & + & & + & + \\
\hline \multicolumn{12}{|l|}{ Prodiamesa sp. } \\
\hline \multicolumn{12}{|l|}{ Orthocladiinae } \\
\hline Brillia bifida & & & & + & & & & & & & \\
\hline Chaetocladius sp. & & & & + & & & & & & & \\
\hline Corynoneura scutellata & & & & + & & & & & & & \\
\hline Corynoneura carriana & & + & & & & & & & & & \\
\hline Cricotopus (Cricotopus) algarum & & + & + & & & & & & & & \\
\hline C. (Cricotopus) bicinctus & & & & + & + & & & + & & + & \\
\hline C. (Cricotopus) cylindraceus & & & & + & + & & & & & + & \\
\hline C. (Isocladius) sylvestris & + & + & & + & + & & + & + & & + & + \\
\hline C. (Cricotopus) trifascia & & & & + & + & & + & & & + & + \\
\hline Eukiefferiella coerulescens & & & & & & & & & + & & \\
\hline Eukiefferiella claripennis & & & & + & & & & & & & \\
\hline Eukiefferiella sp. & & + & & & & & & & & & + \\
\hline \multicolumn{12}{|l|}{ Euryhapsis subviridis } \\
\hline Orthocladius (Orthocladius) clarki & & & & + & & & & + & & + & + \\
\hline Orthocladius (Pogonocladius) consobrinus & & & & + & & & & & & & \\
\hline
\end{tabular}




\begin{tabular}{|c|c|c|c|c|c|c|c|c|c|c|c|}
\hline \multirow{2}{*}{ Chironomidae collected } & \multicolumn{11}{|c|}{ Rivers } \\
\hline & $\mathrm{M}$ & $\mathrm{K}$ & $\mathrm{S}$ & SZ & $\mathrm{Vj}$ & $\mathrm{BC}$ & $\mathrm{T}$ & $\mathrm{A}$ & $\mathrm{J}$ & $\mathrm{V}$ & VS \\
\hline Paratrissocladius excerptus & & & & + & & & & + & & & \\
\hline $\begin{array}{l}\text { Pseudorthocladius (Pseudorthocladius) } \\
\text { curtistylus }\end{array}$ & & & & & & & & & & + & \\
\hline Psectrocladius (Allopsectrocladius) obvius & & & & + & & & & + & & & \\
\hline P. (Allopsectrocladius) gr. dilatatus & + & & & & & & & & & & \\
\hline P. (Psectrocladius) bisetus & & & & & & & & & & & + \\
\hline P. (Psectrocladius) pancratovae & & & & + & & & & & & & \\
\hline P. (Psectrocladius) psilopterus & + & & + & & & & & & & & \\
\hline P. (Psectrocladius) sordidellus & & & & + & & & & & & + & + \\
\hline P. (Psectrocladius) simulans & & & & + & & & & & & & \\
\hline P. (PPsectrocladius) sokolovae & & & & + & & & & & & & \\
\hline Psectrocladius sp. & & & & + & & & + & + & & & \\
\hline Rheocricotopus robacki & & & & + & & & & & & & \\
\hline Rheocricotopus sp. & & & + & & & & & & & & \\
\hline Chironominae & & & & & & & & & & & \\
\hline Tanytarsini & & & & & & & & & & & \\
\hline Cladotanytarsus (Cladotanytarsus) mancus & + & + & & + & + & + & + & + & & + & + \\
\hline Micropsectra sp. & & + & & & & & & & & & \\
\hline Paratanytarsus austriacus & & & & & & & & & + & & + \\
\hline Paratanytarsus sp. & + & + & + & & & & & & & & \\
\hline Rheotanytarsus curtistylus & & & & + & & & + & & & & \\
\hline Rheotanytarsus sp. & & + & & & & & & & & & \\
\hline Stempellinella edwardsi & & & & & & & & & & & + \\
\hline Stempellinella minor Edwards & & & & + & & & & & & + & \\
\hline Stempellina subglabripennis & & & & & & & & & & + & \\
\hline Tanytarsus bathophilus & & & & + & + & & & & & & + \\
\hline Tanytarsus pallidicornis & & & & + & & & & & & & + \\
\hline Tanytarsus excavatus & & & & + & + & + & & + & & & \\
\hline Tanytarsus sp. & & & & & & & & & & & + \\
\hline Chironomini & & & & & & & & & & & \\
\hline Beckidia zabolotzkyi & + & & & & & & & & & & \\
\hline Chironomus (Chironomus) balatonicus & + & + & & & & & & & & & + \\
\hline C. $($ Chironomus $)$ plumosus & + & + & + & + & + & & & & & & + \\
\hline C. (Chironomus) pallidivittatus & + & + & & & & & & & & & \\
\hline C. (Chironomus) riparius & + & & & & & & & & & & \\
\hline $\begin{array}{l}\text { Chironomus (Chironomus) sp. } \\
\text { Cladopelma lateralis }\end{array}$ & + & + & + & + & + & + & + & + & & + & + \\
\hline Cladopelma viridulum & + & & + & & & & & & & & \\
\hline $\begin{array}{l}\text { Cryptochironomus (Cryptochironomus) } \\
\text { psittacinus }\end{array}$ & & & & & + & & & & & & \\
\hline C. (Cryptochironomus) defectus & + & + & + & + & & & & & & + & + \\
\hline C. (Cryptochironomus) obreptans & & & & + & & & & & & + & + \\
\hline C. (Cryptochironomus) albofasciatus & & + & & & & & & & & & \\
\hline C. (Cryptochironomus) psittacinus & & + & & & & & & & & & \\
\hline C. (Cryptochironomus) ussouriensis & + & & & & & & & & & & \\
\hline Cryptotendipes holsatus & & & & + & & & & & & & \\
\hline Cryptotendipes nigronitens & + & & & & & & & & & & \\
\hline Cryptotendipes sp. & + & + & + & & & & & & & & \\
\hline Dicrotendipes modestus & & & & + & & + & & & & + & + \\
\hline Dicrotendipes nervosus & & & & + & & & + & & & + & \\
\hline Dicrotendipes notatus & + & + & + & & + & & & & & + & \\
\hline $\begin{array}{l}\text { Demicryptochironomus } \\
\text { (Demicryptochironomus) vulneratus }\end{array}$ & & & & & & & + & & & + & \\
\hline Einfeldia pagana & & & & & & & & & & & + \\
\hline
\end{tabular}




\begin{tabular}{|c|c|c|c|c|c|c|c|c|c|c|c|}
\hline \multirow{2}{*}{ Chironomidae collected } & \multicolumn{11}{|c|}{ Rivers } \\
\hline & $\mathrm{M}$ & $\mathrm{K}$ & $\mathrm{S}$ & $\mathrm{SZ}$ & $\mathrm{Vj}$ & $\mathrm{BC}$ & $\mathrm{T}$ & A & $\mathrm{J}$ & $\mathrm{V}$ & $\mathrm{VS}$ \\
\hline Endochironomus albipennis & + & + & + & + & & + & + & & & + & + \\
\hline E. tendens & & & & & & + & & & & & + \\
\hline Endochironomus sp. & & & + & & & & & & & & \\
\hline Glyptotendipes (Glyptotendipes) glaucus & + & + & + & + & + & + & & & & + & + \\
\hline G. (Glyptotendipes) cauliginellus & + & + & + & & & & + & & & + & + \\
\hline G. (Glyptotendipes) barbipes & & & & & & & & & & + & \\
\hline G. (Glyptotendipes) paripes & + & + & + & & & & & & & + & \\
\hline Glyptotendipes sp. & + & + & + & & & & & & & + & \\
\hline Harnischia curtilamellata & & & & + & & & & & & & \\
\hline Harnischia sp. & & & & + & & & & & & + & \\
\hline Lipiniella araenicola & + & & & & & & & & & & \\
\hline Lipiniella moderata & & & & + & & & & & & & \\
\hline Microchironomus tener & + & + & + & & & & & & & + & \\
\hline Microtendipes pedellus & & & & + & & & + & & & & + \\
\hline Parachironomus gracilior & + & & & & & & & & & & \\
\hline Parachironomus varus & & & & & & & & & & & \\
\hline Parachironomus pararostratus & & + & & & & & & & & & \\
\hline Paracladopelma camptolabis & + & & & + & & & & & & & \\
\hline Paralauterborniella nigrohalteralis & & & & & + & & & & & & \\
\hline Paratendipes albimanus & + & + & + & + & & & + & + & & & \\
\hline Paratendipes nudisquama & & & & + & & & & & & & \\
\hline Paratendipes sp. & + & & & & & & & & & & \\
\hline Polypedilum (Tripodura) bicrenatum & & & + & + & & & + & & & + & + \\
\hline P. (Uresipedilum) convictum & + & + & + & + & + & & + & & & + & \\
\hline P. (Pentapedilum) exsectum & & + & & + & & & & & & & \\
\hline P. (Polypedilum) nubeculosum & + & + & + & + & + & & + & & & + & + \\
\hline P. (Polypedilum) nubifer & & & & & & & & & & + & \\
\hline P. (Tripodura) scalaenum & + & + & + & + & + & & & & + & + & \\
\hline P. (Pentapedilum) sordens & & & & & & & & & & & \\
\hline Polypedilum (Tripodura) tetracrenatum & + & & & & & & & & & & \\
\hline Polypedilum sp. & + & & + & & & & & & & & \\
\hline Stictochironomus crassiforceps & & & & + & & & & & & & \\
\hline S. rosenschoeldi & & & & + & & & & & & & + \\
\hline Stictochironomus sp. & + & & & & + & & & & & & + \\
\hline Synendotendipes dispar & + & + & + & & & & & & & & \\
\hline S. impar & + & + & + & + & + & & & & & + & + \\
\hline
\end{tabular}

\section{References}

Abakumov, V. 1983. Guidance in methods of the hydrobiological analysis of surface waters and bottom sediments. Gidrometeoizdat, 239 p.

Kondrateva, T.A., Nazarova, L.B. 2011. Dynamic of structural and functional characteristics of chironomid communities from small rivers from areas with steady anthropogenic load. In: Zinchenko T.D., Rozenberg G.S. (Eds.) Ecology of small rivers in 21 century: biodiversity, global changes and denaturalisation of ecosystems. IEVB RAS, Togliatty, p. 67.

Mingazova, N.M. 2012. Catalogue of water objects of Kazan. Volga region. Kazan: Foliant, 131 p.

Nazarova, L.B. 1999. Development of modern views on theratogenic influence of anthropogenic factors on chironomid larvae. $\mathrm{PhD}$ thesis. Kazan University. 150 p.

Nazarova, L. B., Semenov, V. F., Sabirov, R. M., Efimov, I. Yu. 2004. The state of benthic communities and water quality evaluation in the Cheboksary Reservoir. - Water Resources 31 (3): 316-322.

Torsuev, N.P., Mingazova, N.M., Latypova, V.Z., Boyko, V.A. 2005. Ecology of Kazan. Kazan, 527p.

Yakovlev, V.A. 2003. Ecological problems of small rivers of the Republic of Tatarstan (Mesha, Kazanka and Svijaga). Kazan: Fan, 288 p. 